\section{Immunocytochemical evidence for the presence of arginine vasotocin in the rat pineal gland}

THE discovery that the pineal gland contained melatonin' provided the stimulus for a multidisciplinary approach to the study of this organ. Considerable evidence suggests that this gland affects reproductive physiology. For example, it contains biologically active polypeptides. One of the first of these to be identified was arginine vasotocin (AVT), initially isolated from extracts of bovine pineal gland ${ }^{2}$, and later identified chemically and found to have antigonadotropic properties ${ }^{3,4}$. There is physiological and radioimmunoassay evidence for the origin of AVT in the pineal gland. We now present immunocytochemical evidence for the presence of this polypeptide in the pineal gland.

AVT was conjugated to bovine serum albumin, and antiserum to the complex was prepared in adult male guinea pigs. The antiserum was absorbed with $1 \mathrm{mg}$ of bovine serum albumin per $\mathrm{ml}$ of undiluted serum before use. Pineal glands and hypothalami from ten adult male rats were fixed in Bouin's solution and embedded in paraffin. Immunochemical staining was carried out with undiluted antiserum to AVT according to the procedure of Nakane and Pierce? Controls similar to those described by Petrusz $^{8}$ were carried out to assure specificity of staining.

Because of the marked structural similarity of AVT, arginine vasopressin (AVP) and oxytocin, and because other investigators have claimed that the pineal gland contains $\mathrm{AVP}^{6}$ and luteinising hormone releasing hormone (LHRH), establishment of the specificity of the antiserum to AVT was important. The findings summarised in Table 1 strongly suggest that the antiserum to AVT was specific for AVT. Moreover, the supraoptic nucleus, the paraventricular nucleus and the median eminence (regions reputedly rich in AVP, oxytocin, and LHRH, respectively) did not stain with antiserum to AVT, substantiating the immunocytochemical specificity of the antiserum to AVT.

Antiserum to AVT immunochemically stained a distinct population of cells in the rat pineal gland (Fig. 1). These cells were distributed diffusely throughout the gland. They were very irregular in form with extensive perivascular and intercellular processes. Cytoplasmic processes were frequently cupped around neighbouring cells. Most parenchymal cells were regular in outline and were identified as cells clasically described as pinealocytes or chief cells. Their characteristic pale nuclei with infolded nuclear membranes

Fig. 1 Light micrograph of a section from a rat pineal gland immunochemically stained with antiserum to AVT. The darkly staining reaction product of the Nakane-Pierce technique defines the presumptive AVT cells.

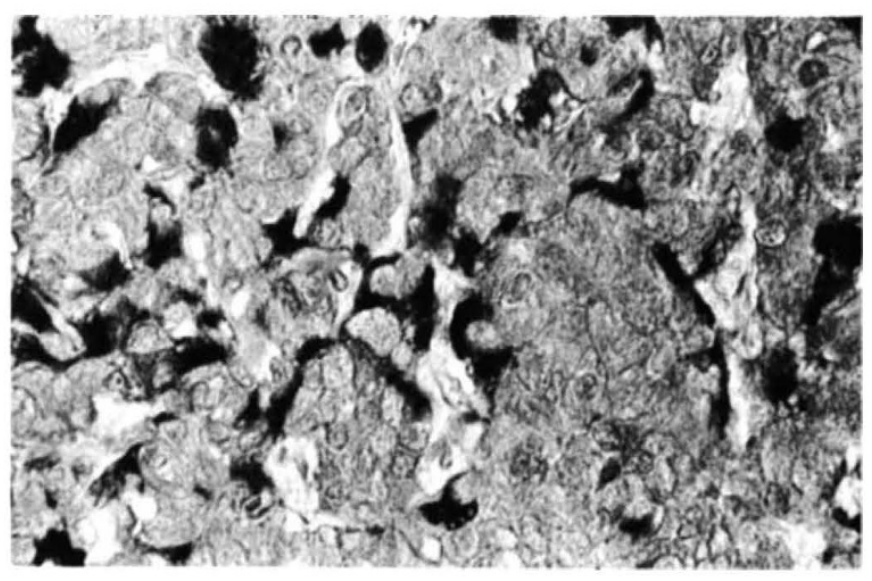

Table 1 Immunological specificity of antiserum to AVT

Anti-AVT Anti-AVT Anti-AVT Anti-AVT Anti-AVT

$\stackrel{+}{A V T} \stackrel{+}{A V P} \stackrel{+}{O} \quad \stackrel{+}{\text { LHRH }}$

Pineal

gland

$-$

$+$

$+$

$+$

Immunological specificity was tested by absorbing undiluted antiserum to AVT with $0.2 \mathrm{mg}$ of AVT, AVP, OT, or LHRH, before immunochemical staining of rat pineal sections.

and many nucleoli were in marked contrast to the intensely staining, elongate nuclei of the immunochemically stained cells. The pronounced staining of the nuclei of the presumptive AVT cells was interesting because this was not normally observed after immunocytochemical staining of cells containing polypeptides.

These findings add further evidence for the presence of AVT in the pineal gland. Whether the immunocytochemically stained cells of this study represent a second line of pinealocytes or are glial in origin, and whether they synthesise or store AVT has yet to be determined.

We thank Dr E. G. Rennels for helpful criticism and Dr M. K. Vaughan for encouragement.

\section{E. P. Bowie \\ D. C. Herbert}

Department of Anatomy,

University of Texas Health Science Center,

San Antonio, Texas 78284

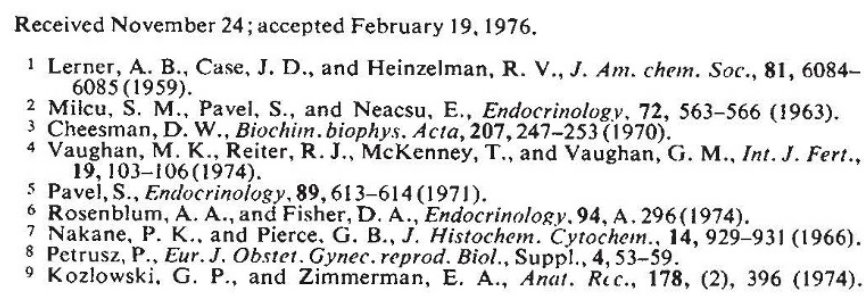

\section{Functional roles of luminal sodium and potassium in water transport across desert scorpion ileum}

IN terrestrial insects the rectum is a major site of fluid absorption and is, therefore, important in the physiological control of water balance. Water absorption in the insect rectum occurs against osmotic gradients greater than 300 mosmol $1^{-1}$ and apparently in the absence of net ion movements from lumen to haemolymph ${ }^{1,2}$. Evidence suggests that net water fiow from the rectal Jumen to haemolymph results from increases in intercellular space osmotic pressure due to the transport across lateral cell membranes of recyclable intracellular solutes ${ }^{3,4}$. Little is known about the mechanism of water transport in the insect gut anterior to the rectum nor about gastrointestinal processes involved in water regulation in other terrestrial arthropods. Scorpions are ideal organisms for measurements of arthropod ileal transport because this part of the gut extends nearly the entire length of the tail and can be isolated for in vitro perfusion. We present here preliminary information on a sodium-dependent, potassium-inhibited water transport mechanism in the ileum of the desert scorpion Hadrurus arizonensis.

Scorpions were anaesthetised lightly with chloroform, and the eritire ileum $(2 \mathrm{~cm})$ was removed and placed in a saline medium. The ionic composition and osmotic pressure of the medium were based on data obtained from flame photometry, chloride titration and osmometry of haemolymph samples, as well as on values reported from other scorpion species in the literature ${ }^{5-7}$. This scorpion saline had an osmotic pressure 